\title{
Streamer Branching in a Short Gap: The Influence of the Power Supply
}

\author{
E. M. van Veldhuizen, P. C. M. Kemps, and W. R. Rutgers
}

\begin{abstract}
The formation of streamers in a $25-\mathbf{m m}$ gap in air is studied with an intensified charge coupled device camera with high resolution in space and time. Strong branching is observed and streamers reach the cathode in an area that is much wider than the gap length. Switching the high voltage with either a spark gap or a semiconductor stack has a large influence on the branching: much more discharge channels appear when using the semiconductor switch. Pictures taken with 0.8-ns resolution show that the streamers propapagate with $3 \mathrm{~ms} / \mathrm{mm}$ near the electrodes and with $0.5 \mathrm{~mm} / \mathrm{ns}$ in the middle of the gap.
\end{abstract}

Index Terms-Corona, gas discharges, MOSFET switches, optical imaging.

D ISCHARGES in ambient air at a pressure of 1 bar are used nowadays for many purposes [1]. The shape of these discharges is very complex: the streamers branch in three-dimensional (3-D) shapes. Numerical studies mostly use an approach with branching probabilities [2]. This method is still in use for the description of dielectric breakdown in liquids and solids [3], and lightning [4]. A first approach to simulate branching from physical processes is given in [5].

The available models do not (yet) give insight to the banching phenomena. Therefore, the branching of streamers is studied experimentally using a fast charge coupled device (CCD) camera. This investigation includes the influence of the power supply.

The measurements are performed in a 25-mm gap in ambient air. The gap configuration is point-to-wire with the wire perpendicular to the viewing direction of the camera to get the streamers in focus. The corona discharge is created by a pulsed power supply which is a charged capacitor of $1 \mathrm{nF}$, and a fast switch. Two switches are available: a spark gap and a semiconductor switch. The spark gap is standard in this type of circuit, but its disadvantages are large jitter, difficult adjustment, and limited lifetime. The semiconductor switch (Behlke HTS-301) is a stack of high-voltage MOSFET transistors and it can switch up to $30 \mathrm{kV}$ with a jitter of $0.1 \mathrm{~ns}$. The camera used to observe the streamers is a CCD with image intensifier (Andor Technology DH534-18). Its main specifications are: $1024 \times 1024$ pixels, sensitivity up to photon counting, minimum optical gate $0.8 \mathrm{~ns}$, and spatial resolution $21 \mu \mathrm{m}$. The camera is equipped with a high-quality quartz lens (Nikon UV-Nikkor $105 \mathrm{~mm} \mathrm{f} / 4.5$ ) to capture the $\mathrm{N}_{2}$-SPS emission that dominates the spectrum. A delay generator (Stanford D6535) is used to synchronize the discharge and the camera.

Manuscript received April 27, 2001

The authors are with the Department of Physics, Technische Universiteit Eindhoven, Eindhoven 5600 MB, The Netherlands (e-mail: e.m.v.veldhuizen@tue.nl).

Publisher Item Identifier S 0093-3813(02)03328-3.
Fig. 1 shows three pictures with illumination times longer than the time required by the streamers to cross the gap. The semiconductor switch is used here and the rise time of the voltage pulse is $40 \mathrm{~ns}$. Fig. 1(a) is at $6 \mathrm{kV}$ which is just above inception, Fig. 1(c) is at $25 \mathrm{kV}$ which is just below breakdown and Fig. 1(b) is at $12.5 \mathrm{kV}$, an arbitrary intermediate level. At $6 \mathrm{kV}$, only one filament is observed which travels from the anode several millimeters toward the cathode. At the higher voltages, the streamers bridge the gap and a lot of branches are observed.

Fig. 2(a) shows a picture taken using the spark gap as switch creating a $25-\mathrm{kV}$ voltage pulse with a rise time of $30 \mathrm{~ns}$. The number of streamers and branches is much less than in Fig. 1(c). The streamers appear thicker, but this is an artefact caused by the way the picture is made. When the full-width at half-maximum (FWHM) of the channels is measured, it turns out that all streamers have diameters in the range of 150-200 $\mu \mathrm{m}$ [6]. The energy input per pulse is $3 \mathrm{~mJ}$ in the case of Fig. 1(c) and $1 \mathrm{~mJ}$ for Fig. 2(a).

To check the influence of the risetime, some additional resistance $(1 \mathrm{k} \Omega)$ and inductance $(\sim 1 \mu \mathrm{H})$ is added to the circuit. This influence is shown in Figs. 2(b) and (c), respectively. These are taken at the same conditions as Fig. 1(b). One sees that the streamers have a somewhat lower intensity and propagate not completely to the anode.

Fig. 3 shows a sequence of pictures taken with the shortest opening time of the image intensifier, $0.8 \mathrm{~ns}$ at a voltage of $25 \mathrm{kV}$ using the semiconductor switch. The discharge starts similar to Fig. 1(a), but the streamer diameter is larger. At 9 ns, this filament explodes into separate streamer heads. The number of streamers heads is much higher than in the case of a spark gap (see pictures in [6]). The length of the streamer heads shows that the propagation velocity is $\sim 3 \mathrm{~mm} / \mathrm{ns}$ when the streamers move away from the anode [Figs. 3(b) and (c)] and just when the first streamer hits the cathode [Fig. $3(\mathrm{~g})]$. The velocity in the middle of the gap is considerably lower, $\sim 0.5 \mathrm{~mm} / \mathrm{ns}$ [Fig. 3(f)]. The main reason for this effect is probably the local electric field strength.

The difference in the numbers of streamers between spark gap and the semiconductor switch cannot be explained from a difference in resistance or inductance of the switches considering their small influence as shown in Figs. 2(b) and 2(c). The capacitance of the switches is also quite different. This is to be investigated. The pictures shown here do not really resemble the results of the stochastic models. Further study is required since branching can be expected to be very important for the effectiveness of corona discharges in applications. 




Fig. 1. Time integrated pictures of streamers in a $25 \mathrm{~mm}$, point-to-wire gap in ambient air at (a) $6 \mathrm{kV}$, (b) $12.5 \mathrm{kV}$, and (c) $25 \mathrm{kV}$. A semiconductor switch is used to create the voltage pulse for these photographs.



Fig. 2. Picture (a) is taken with a $25 \mathrm{kV}$ pulse created with a spark gap. Pictures (b) and (c) are taken using the semiconductor switch at $12.5 \mathrm{kV}$ with additional resistance and inductance resp.



Fig. 3. Pictures of streamers taken with illumination times of $0.8 \mathrm{~ns}$. The delays of the frames with respect to discharge initiation (see text) are: (a) $3 \mathrm{~ns}$, (b) $5 \mathrm{~ns}$, (c) $9 \mathrm{~ns}$, (d) $16 \mathrm{~ns}$, (e) $31 \mathrm{~ns}$, (f) $51 \mathrm{~ns}$, and (g) $71 \mathrm{~ns}$.

\section{REFERENCES}

[1] E. M. van Veldhuizen, Ed., Electrical Discharges for Environmental Purposes: Fundamentals and Applications. New York: Nova Science, 1999, ISBN 1-56 072-743-8.

[2] S. Badaloni and I. Gallimberti, "Monte Carlo simulation of streamer branching," in Proc. ICPIG XI, Prague, Czech Republic, 1973, p. 196.

[3] H. A. Fowler, J. E. Devaney, J. G. Hagedorn, and F. E. Sullivan, "Dialectric breakdown in a simplified parallel model," Comput. Phys., vol. 12, pp. 478-487, 1998.

[4] A. L. Kupershtock, V. Charalambakos, D. Agoris, and D. I. Karpov, "Simulation of breakdown in air using cellular automata with streamer to leader transition," J. Phys. D, Appl. Phys., vol. 34, pp. 936-946, 2001.
[5] I. P. Vereshcagin, A. A. Beloglovsky, V. N. Vinokurov, O. I. Kondratov, L. L. Chernenskyy, and E. J. M. van Heesch, "Mathematical model of impulse streamer corona from a spherical electrode," in Proc. XIII Int. Conf. on Gas Discharges and their Applications., S. J. MacGregor, Ed., Glasgow, U.K., Sept. 3-8, 2000, p. 575.

[6] E. M. vanVeldhuizen, A. H. F. M. Baede, D. Hayashi, and W. R. Rutgers. Fast imaging of streamer propagation. presented at Proc. APP Spring Meeting. [Online]. Available: http://www.ilp.physik.uni-essen.de/doebele/Spring2001/pdf/116.pdf 